\title{
Depth-based Adaptive and Energy-aware (DAE) Routing Scheme for UWSNs
}

\author{
Saqib Shahid Rahim ${ }^{1,2,3}$, Sheeraz Ahmed ${ }^{2,3}$, Fazle Hadi ${ }^{2}$, Ayub Khan²,3, M.Usman Akhtar ${ }^{4}$, Lailma \\ Javed $^{5}$
}

1Abasyn University, Peshawar, Pakistan 2Preston University, Peshawar, Pakistan 3Career Dynamics Research Centre, Peshawar, Pakistan 4University of Engineering and Technology, Peshawar, Pakistan 4University of Engineering and Technology, Peshawar, Pakistan

5COMSATS Institute of Information Technology, Attock Campus, Pakistan

1saqib.shahid@abasyn.edu.pk, 2sheerazahmed306@gmail.com,3fhadi76@yahoo.com, 4ayub2060@gmail.com,

5lasolutionen@gmail.com, 6lailma_javed@ ciit-attock.edu.pk 3

\begin{abstract}

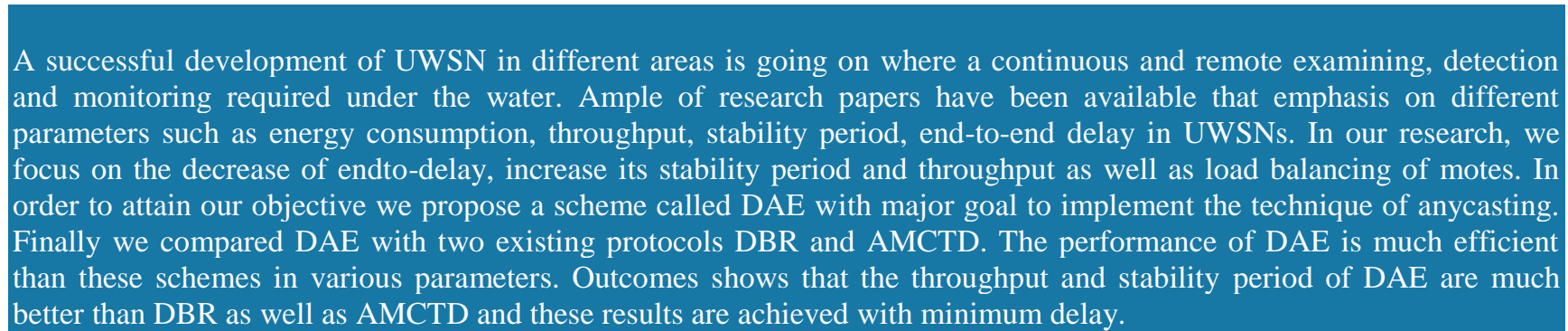

Keywords: Stability Period, UWSN, Throughput, End-to-End Delay, Anycasting.

Received on 03 September 2017, accepted on 05 January 2018, published on 10 April 2018

Copyright (C) 2018 Saqib Shahid Rahim et al., licensed to EAI. This is an open access article distributed under the terms of the Creative Commons Attribution licence (http://creativecommons.org/licenses/by/3.0/), which permits unlimited use, distribution and reproduction in any medium so long as the original work is properly cited.

doi: 10.4108/eai.10-4-2018.154449

\section{Introduction}

Our planet earth consists of about $70 \%$ water and an interesting thing is that just $10 \%$ of it is investigated which means that a huge area is not explored. Various issues are exists in the customary methods to examine oceanic environment like expensiveness and require lengthy time to get results of water examining hence the UWSNs are significant option for examining oceanic environs [1]. UWSN set up under the water and an excessive growing technology for exploring and examining massive un-explored underwater applications [2]. A basic architecture of UWSN includes multiple immovable motes to examine a particular region. The gathered information is forwards to one or more $\operatorname{sink}(\mathrm{s})$. Motes links are either preplanned or remotely allocate by the user. To manually constitute number of motes links, a centralize system needs extensive effort. Moreover in this situation reconfiguration of links also require in case if one or more mote(s) added or died [3]. Usually sink has greater energy than other motes that have limited powered battery [4]. Each mote fortified with sensors and an acoustic modem that have low bandwidth to communicate with sink and others motes. The consumption of energy of an acoustic modem and a common radio modem is not same [5]. Sinks used in UWSNs also known as sonobuoys that exist at the surface of water. Each sink is also fortified with two modems; one is acoustic modem to communicate with motes under the water and a radio modem to communicate with onshore station or satellite. 
All motes in a Sensor Equipped Aquatic (SEA) Swarm, monitors their neighboring underwater activities and using any casting transmits significant information to one of sonobuoys/sink with acoustic multi hop routing. Received information then unloaded to an examining point using radio waves for auxiliary off-line usage [6]. A simple architecture of UWSN is shown in figure 1.

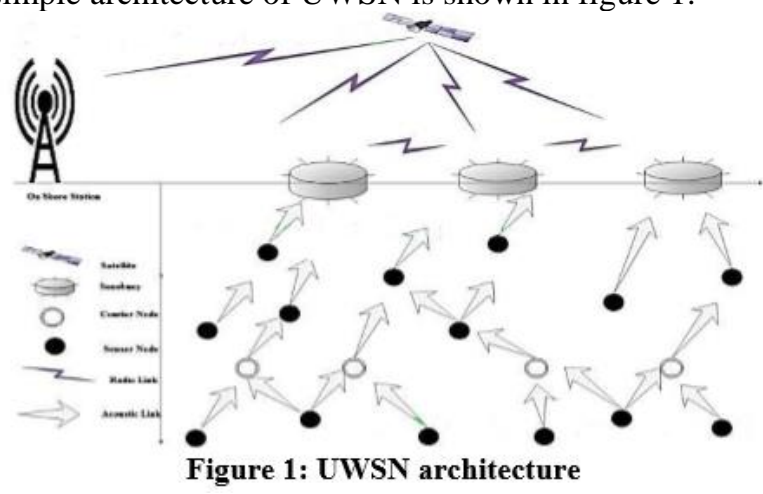

A UWSN sink is either static or mobile. In case of static, motes that near to surface sink consume more energy as compare to other hence the network life minimizes. Moreover for balance of sensor nodes energy consumption, researchers also introduced schemes that have the concept of mobile sensor nodes in which the neighbors of static sink is rapidly changes. The other case where sink is mobile also exists but it is not fit for delay sensitive cases [7]. The data gathering of UWSNs is increases by mobile motes or sinks hence to maximize the data collection many schemes nowadays deploying mobile sensors and/or mobile sinks but the cost of these nodes and sink deployment is high and difficult to handle, due to this many application not use it [8]. Nowadays UASN is a developing research field that advances numerous applications in underwater research [6]. UWSN provides a number of applications such as pollution detection, ocean current detection, discovery of submarines, under the ocean surveys and disaster avoidance [4], [6]. Hence that is the main reason behind the attraction of researchers to UWSN area [5]. UWSN use sound or acoustic waves as communication medium instead of radio and optical due to their low performance as radio degrade in underwater easily and rapidly. Hence the UWSNs used acoustic signal as communication medium and that's why, most of the researchers call it Under Water Acoustic Sensor Networks (UWASNs) too [2]. Radio signals transmit data to extensive distances at very low frequencies that is 30 to $300 \mathrm{~Hz}$, and requires big antennas and extraordinary power. Moreover radio waves rapidly absorb in water hence this medium not offer requisite bandwidth and range. Similarly optical waves are also not suitable as these highly affect from scattering and require great precision in pointing narrow beam of laser [9][10]. Hence the acoustic or sound waves are appropriate medium of data transmission in the environment of underwater as the alternate of optical or radio. Rest of the paper is further divided into sub sections which are describe as follow. Section II provides some existed schemes. In section III motivation behind this research is mentioned. Proposed model for DAE protocol is presented in Sections IV. Simulation outcomes discussed in $\mathrm{V}$ section and finally paper is concluded in section VI.

\section{Related work}

Different researchers proposed different protocols and techniques to increase performance and reliable communication. Some of them are summarized and discussed below. LEACH [11] is the hierarchical protocol that based on clustering for identical WSNs. The major objective is minimizing global communications by establishing local cluster of motes that based on least either received strength of signal or distance. For every cluster, the cluster head $(\mathrm{CH})$ is selected, that is used for data collection and merging. After that $\mathrm{CH}$ sends data further to base station (BS), thus saving energy as number of transmissions and distance is condensed. Sink and BS interchangeably used for that service. Researchers in [12] proposed a routing protocol that is capable to respond instantly after revealing of alteration in sensed characteristic of interest which is reactive approach. Election of $\mathrm{CH}$ and nodes association technique is identical, but the dissimilarity comes in the transmission of data transmission in [11] technique. However, in [12] there is check based on soft and hard threshold transmission. This eventually results in reduced network throughput. Depending on heterogeneity of nodes, the SEP [13] describes two levels of energy. Based on these levels the motes are categorized in to normal and advance motes. The advance motes exist $\alpha$ time additional energy as compare to normal. Hence advanced nodes preferred more for the $\mathrm{CHs}$ because of their probability weights that are assigned. In [14] the authors proposed DEEC scheme for heterogeneous WSNs. In DEEC the nodes are fortified with dissimilar levels of energy. The $\mathrm{CH}$ election is depending on the residual energy of nodes. The nodes that have greater residual energy have greater chances to be $\mathrm{CHs}$ for a specific round. Due to this among nodes a uniform energy distributes. The $\mathrm{CH}$ selection in [14] is same as in [11], and the possibility of nodes to select $\mathrm{CHs}$ is not same. Structure for enhancing the network lifetime by employing mobility of sink in applications that tolerate delayed data delivery to the sink is presented in [15]. The Authors of this scheme expressed optimization issue to increase the network lifetime, subject to constraints of delay bound, flow conservation and energy. In [16], authors proposed mutual sink mobility in two level network that consist of advanced and normal nodes. Two sinks are mutually moving in a pre-planned configuration for assembling information. Nodes of network send data to MS directly, when they comes in the range of transmission in delay tolerant mode. To save the energy, idea of sleep and a wake mode is introduced. Limiting 
the movement of sink to restricted number of locations, in [17] researchers created a framework for mutual sink mobility. It is additionally reduced into sub problems due to NP-hardness of the problem. For the solution of sub problem involving single sink, an efficient primal dual algorithm is introduced. In [18] authors presented a mobile information collector placement technique to extend the network life time. Placement issue expressed as MILPs and its solver is used to search the nearby optimum placement and routing paths to deliver information. For WSN researchers in [19] have proposed a scheme with more than one sinks that are mobile in nature. The strategy of Reliable Energy-Efficient Data Dissemination (REDD) is in a specific style that using geographical forwarding, MS directly communicates with source by finding its location. In [20] authors presented another scheme that is biased adaptive sink mobility. This technique depends on the conditions of local network like surrounding density and residual energy. The adaptive mobility is defined according to that sink moves probabilistically in the zone less visited for covering the whole network field in minimum time and adaptively stopping in the zone of high density for collecting maximum amount of information. DBR [21] was a location free technique for routing in UWSNs. In DBR motes use their information about depth for transferring the gathered information to the sink on surface. The motes that have smaller depth contribute in transferring the packets. For each packet mote has specific holding time. Motes that have smaller depth have short holding time while motes that have longer depth have higher holding time. To enhance the efficiency of energy of UWSNs another routing technique that is Energy Efficient Depth Based Routing (EEDBR) [22] is proposed. It is important to note that increasing energy efficiency in UWSNs is one of major and critical issue, due to the battery replacing of UWSNs motes is very expensive as well as difficult as the environ of underwater is harsh. In this regard, authors presented EEDBR scheme for UWSNs. This technique uses two routing matric, depth as well as the residual energy of motes too. Similar to the technique DBR, EEDBR also not require any localization information of motes. In [23], authors proposed another interesting idea which is the use of Acoustic Underwater Vehicles (AUVs) that reduce end-to-end delay. The use of AUVs specially aimed for the UWSNs applications which are delay-sensitive. Direct and multi hop transmission both used in this technique. Similar to [23], in [24] authors presented a totally different idea for the UWSNs in which they use some nodes that have mobility known by the name of courier nodes to increase the network life time. The improved Adaptive Mobility of Courier nodes in Threshold-optimized Depth-based-routing (iAMCTD) enhance the throughput of network. iAMCTD use Forwarding Functions (FFs) that mostly reduces the packet drop ratio.

\section{Motivation}

According to the literature, preponderance of researchers [21],[22] used depth of the sensor nodes as a metric for forwarding data packets but there subsists lack of proper load balancing and by this uneven distribution of load of motes. Hence efficient energy consumption is not appropriately reduced by inappropriate balancing of load among UWSNs motes. [21] takes the only parameter that is depth of motes to forward data. DBR tries to achieve lengthier lifetime of UWSN however the stability period finishes soon by redundant forwarding of data and abundant load on motes that have low depth in the network. [22] technique is based on receiver in which every mote that has lesser depth selects as forwarder. However redundancy of packets during the transmission takes much energy. EEDBR not a cooperative scheme hence packets are sent from source motes to endpoint using an only link but in multi hop manner. By noise and multipath fading environ of UWSN, most of the time the signals undergoes high bit error rate (BER). To overcome the limitation in above discussion we proposed DAE scheme for UWSNs. In this scheme, the performance is increased by taking care of delivery ratio, stability period and end-end-delay.

\section{DAE Proposed Protocol}

This section presents our proposed routing protocol DAE for UWSN with AnyCasting technique to improve the performance of existing schemes.

\subsection{Network Topology}

In UWSN the deployment of sinks, motes and courier nodes is a challenging job and if they are not deploy in proper location it seriously affect the overall performance of network. At the start of this phase eight surface sinks deployed on the surface of water while two hundred twenty five sensor nodes under the water in a pattern from top to the oceanic bed and four courier nodes. It is supposed that the surface sinks fortified with the radio and acoustic modems. The acoustic modems are for communication with the underwater nodes and radio modems for communication with other sinks or data station at ground. Four courier nodes persist at equal horizontal distances from one another while initiates vertical movement to sea bed at initialization of network.

\subsection{Initialization Phase}

For initialization of network all sinks broadcast a "Hello" message to all motes of UW network that contains id of sinks with their locations. On receiving this message, motes save the location and Id of sinks in their routing table. Our approach forwards data receiver-based due to lack of location information. This broadcasted data consist of the node Id, energy and depth. In this way each 
mote aware about their neighbors and sinks, and sinks about nodes. The courier nodes plan their mobility pattern at initialization of UW network and starts gathering packets from source motes.

\subsection{Channel Model}

Following is the channel model that use for computing spread and noise loss in under the water. We also compute signal attenuation from the spreading loss and thorps model for signal absorption loss.

\subsubsection{Absorption Loss and Attenuation}

The channel model computes absorption loss $\alpha(f)$ at a given $\mathrm{f}$ as following [4][6].

The channel model computes absorption loss $\alpha(\mathrm{f})$ at a given $\mathrm{f}$ as following [4][6].

$$
\begin{aligned}
& 10 \log (\alpha(\mathrm{f}))=\frac{0.11 \mathrm{f}^{2}}{\left(1+\mathrm{f}^{2}\right)}+\frac{44 \mathrm{f}^{2}}{\left(4100+\mathrm{f}^{2}\right)}+\frac{2.75 \mathrm{f}^{2}}{10^{4}}+ \\
& 0.003 \text { for } \mathrm{f} \geq 0.4 \\
& \ldots \ldots \ldots . .(1) \quad \text { and } \\
& 10 \log (\alpha(\mathrm{f}))=0.002+0.11\left(\frac{\mathrm{f}}{(1+\mathrm{f})}\right)+ \\
& 0.011 \mathrm{f} \text { for } \quad \mathrm{f}<0.4
\end{aligned}
$$

We calculate total attenuation by combine the spreading and absorption loss as follows

$$
10 \log (\mathrm{A}(\mathrm{l}, \mathrm{f}))=\mathrm{K} * 10 \log (\mathrm{l})+\mathrm{l} * 10 \log (\alpha(\mathrm{f})) \ldots
$$

In (3), 1st term represents spreading loss while the next one absorption loss and $\mathrm{K}$ is spreading coefficient that's commonly used value is 1 for cylindrical, 1.5 for practical spreading and 2 for spherical [4].

In our scheme we calculate the transmission loss by following equation.

$$
\mathrm{TL}=10 * \log (\mathrm{Tx} \mathrm{Rg})+(\mathrm{A}(\mathrm{l}, \mathrm{f}) * \mathrm{TxRg} * 0.001) . .
$$

where $\mathrm{TL}$ is transmission loss, $\mathrm{Tx} \mathrm{Rg}$ is range of transmission and $\mathrm{A}(\mathrm{l}, \mathrm{f})$ is thorp attenuation.

\subsubsection{Noise}

The total noise loss (N(f) ) for a given frequency $f$ can be calculated by adding all types of noises (turbulence, shipping, wave and thermal noise) as [25] [26].

$$
N(f)=N_{t}(f)+N_{s}(f)+N_{\omega}(f)+N_{t h}(f)
$$

These noises $\mathrm{N}_{\mathrm{t}}, \mathrm{N}_{\mathrm{s}}, \mathrm{N}_{\omega}$ and $\mathrm{N}_{\mathrm{th}}$ are formulated respectively as follows

$$
\begin{aligned}
& 10 \log N_{t}(f)=17-30 \log f \ldots \ldots \ldots \ldots \ldots \ldots \ldots(6) \\
& 10 \log N_{s}(f)=40+20(s-0.5)+26 \log f-60 \log (f+0.03) \ldots
\end{aligned}
$$

In (7) $s$ is the shipping factor which lies between zero and one for which are respectively for low and high activities.

$$
10 \log N_{\omega}(f)=50+7.5 \omega^{1 / 2}+20 \log f-40 \log (f+0.4)
$$

where $\omega$ is speed of wind that is in $\mathrm{m} / \mathrm{s}$.

$10 \log \mathrm{N}_{\text {th }}(\mathrm{f})=-15+20 \log \mathrm{f}$

\subsubsection{SNR}

SNR in Underwater Channels for narrowband with frequency f stated as

$$
\operatorname{SNR}(\mathrm{d}, \mathrm{f})=\frac{\mathrm{Pw}}{\mathrm{A}(\mathrm{d}, \mathrm{f}) \mathrm{N}(\mathrm{f}) \mathrm{Bw}}
$$

In (10) pw is power of transmission in watts, $\mathrm{Bw}$ is bandwidth in $\mathrm{Hz}$ received at distance d [6].

\subsection{First order energy model}

The energy dissipation in mode of transmits and receives change the outcomes of several schemes. In our technique, we assumes a model where radio dissipates $\mathrm{E}_{\text {elec }}=50 \mathrm{~nJ} / \mathrm{bit}$ to run the circuitry of receiver and transmitter while eamp $=100 \mathrm{pJ} / \mathrm{bit} / \mathrm{m} 2$ for transmit amplifier. Hence for transmission of $\mathrm{k}$ bit message a distance $\mathrm{d}$ by using our model.

$$
\mathrm{E}_{\mathrm{TX}}(\mathrm{k}, \mathrm{d})=\mathrm{E}_{\mathrm{Tx}-\mathrm{elec}}(\mathrm{k})+\mathrm{E}_{\mathrm{TX}-\mathrm{amp}}(\mathrm{k}, \mathrm{d})
$$

1)

$\mathrm{E}_{\mathrm{TX}}(\mathrm{k}, \mathrm{d})=\mathrm{E}_{\text {elec }} * \mathrm{k}+\varepsilon_{\mathrm{amp}} * \mathrm{k} * \mathrm{~d}^{2}$.

and for receiving that message,

$$
\begin{aligned}
& \mathrm{E}_{\mathrm{Rx}}(\mathrm{k})=\mathrm{E}_{\mathrm{Rx}-\text { elec }}(\mathrm{k}) \\
& \mathrm{E}_{\mathrm{Rx}}(\mathrm{k})=\mathrm{E}_{\text {elec }} * \mathrm{k} \ldots
\end{aligned}
$$

$E_{T x}$ is energy required for the transmission while $E_{R x}$ energy required receiving bits by receiver. The $\mathrm{k}$ denotes bits size, $d$ is distance between nodes and sinks. $\mathrm{E}_{\mathrm{Tx}-\mathrm{elec}}$ and $\mathrm{E}_{\mathrm{Rx} \text {-elec }}$ is the energy that is required for working of electronic circuitry of the receiver and transmitter to receive and transmit bits. $\varepsilon_{\mathrm{amp}}$ is the energy required to amplify $\mathrm{k}$ bits to the distance $\mathrm{d}$.

\subsection{Cost Function Computation}


Our scheme calculates a cost function at each neighbor mote for forwarder node (ni) selection. Condition of selection for a node as a forwarder is having maximum value of CF. One of a cost function used by DAE scheme are as follow

$$
\begin{aligned}
& \mathrm{LdCFi}=\mathrm{REi} /(\mathrm{PL}(\mathrm{d}, \mathrm{f}) * \mathrm{Di}) \\
& \mathrm{MdCFi}=(\mathrm{PL}(\mathrm{d}, \mathrm{f}) * \mathrm{Di}) / \mathrm{REi} \\
& \mathrm{HdCFi}=(\mathrm{PL}(\mathrm{d}, \mathrm{f}) * \mathrm{REi}) / \mathrm{Di}
\end{aligned}
$$

where $\mathrm{LdCF}$ is low depth cost function, $\mathrm{MdCF}$ is medium depth cost function, $\mathrm{HdCF}$ is high depth cost function. The PL is path loss, RE is residual energy D is the depth of node. If the forwarder is at low depth, it use LdCF or if at medium depth, use MdCF and if at high depth, use HdCF.

\subsection{Distance Computation for AnyCasting}

When the data starts travelling from the bottom of the water and reaches the top most layer of the network, the nodes then checked whether the next hop is forwarding nodes or the sink. As there are multiple sinks deployed on water surface, hence anycasting needs to be employed at this stage. This will give us two profound achievements. Firstly load balancing will be achieved at the top most layer of the network which is normally overburden due to data aggregation from lower layers as well as data routing. Secondly the data delivery will become quicker if nearest sink is selected for final data sending.

For to apply this technique, every node which has the sink as the next hop will compute its distance from the all sinks available using distance formula as given below.

$$
\mathrm{dt}=\sqrt{\left(\mathrm{p}_{2}-\mathrm{p}_{1}\right)^{2}+\left(\mathrm{q}_{2}-\mathrm{q}_{1}\right)^{2}}
$$

In (18) dt denotes distance between node to sink, $\mathrm{p}_{2}$ and $\mathrm{q}_{2}$ are node location while $\mathrm{p}_{1}$ and $\mathrm{q}_{1}$ are sink location. Least value of the distance formulae will help select the nearest sink and only one sink at a time for final data delivery and hence the concept of anycasing plays its role for efficient data routing.

\subsection{Data Transfer}

When the data is reached at the best one sink which is nearest to the forwarder node, this collected data is transfer to the base station that is situated at onshore of the ocean. This transmission is done by radio signals instead of acoustic. In some cases collected data transfer to satellite from surface sinks to transmit it further along distance.

\section{Results and Discussion}

In this paper a chain of simulations shown to test the efficiency of our DAE scheme. We compared DAE scheme with two well-known schemes: DBR and AMCTD.

\subsection{Stability Period}

The operational time of network until $1^{\text {st }}$ mote expires is called stability period. Figure 4 shown the performance of DAE technique that is optimal than DBR and AMCTD. At second 10000 DAE loses 102 nodes and 123 nodes are alive while AMCTD and DBR lose 140 and 178 nodes respectively. The improved performance of DAE is because of nodes balanced usage of energy.

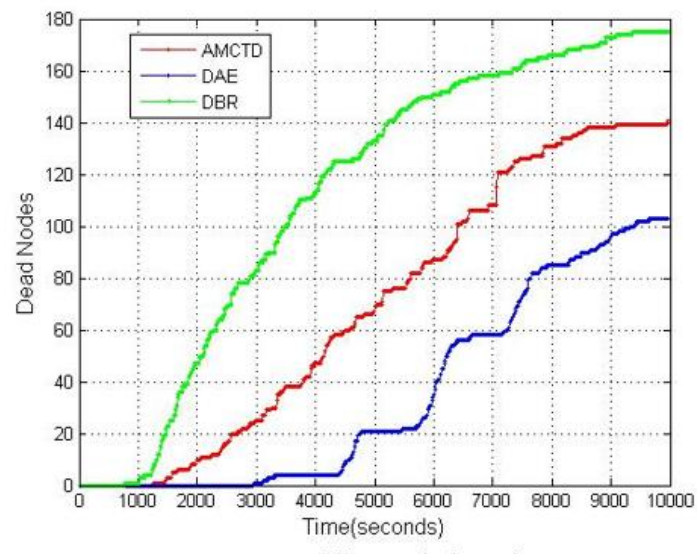

Figure 2: Stability Period vs Time

\subsection{End-to-End Delay}

End to end delay states the lag within source mote and terminus sinks. Reducing end to end delay is a significant feature to be considered in UWSN. Detected data of sensor nodes analyzed at $\mathrm{BS}$, hence received data required exactly on time. Figure 4 depicts that DAE performance is improved than AMCTD and DBR. Figure shows that delay of DAE is 35 at 1000 second and 5 at 10000 seconds while AMCTD and DBR showing 92 to 28 and 50 to 5 from 1000 to 10000 seconds. The achieved performance is due to anycasting among sinks at surface. Availability of more than one surface sink in DAE attains performance improved as every surface sink collect limited of nodes data.

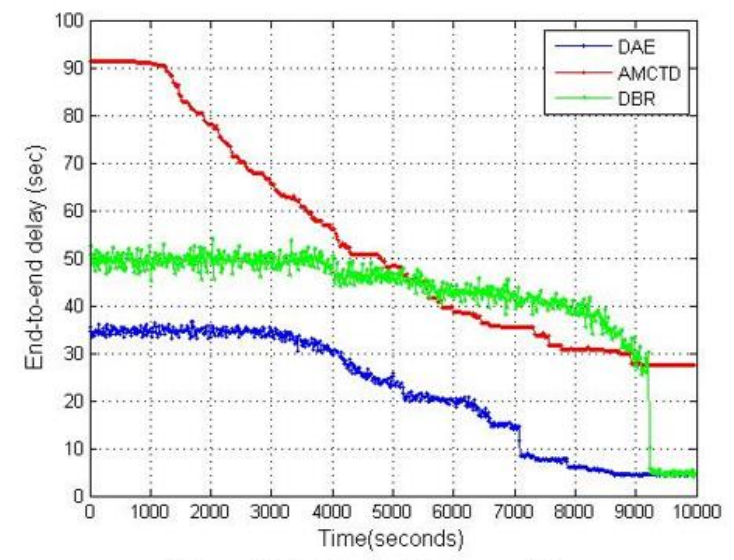

Figure 3: End-to-End Delay vs Time

\subsection{Throughput}

Throughput is also known as Packet Delivery Ratio (PDR) which is effective delivery of data from nodes to surface sinks. The links used in DAE are of two types that are from sensor node to forwarder and forwarder to 
surface sinks. Second one type of link transfer most data than the first one. Hence, in DAE mostly sensors come close to the surface sink. It transfers data packets directly, that becomes the reason to improve throughput. Results of figure 6 depict a perfect difference in efficacy among DAE, AMCTD and DBR schemes. Figure depicts that DAE is well than of AMCTD and DBR. The results proved that the throughput of DAE is showing stable PDR which is clearly better than AMCTD and DBR that are declining with increasing of time.

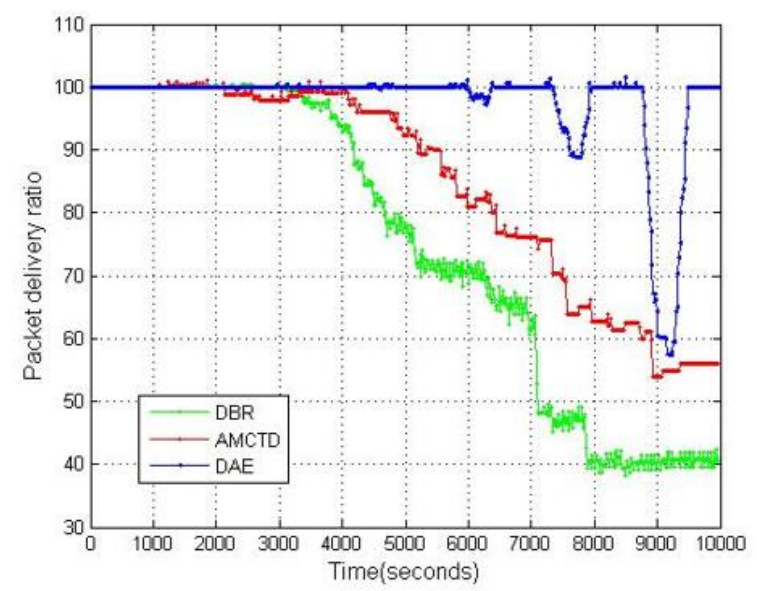

Figure 4: Throughput vs Time

\subsection{Net Transmission Loss}

Transmission loss is also known as path loss that is loss of data during the transmission. According to the figure 5 it is proved that transmission loss is much lower than AMCTD and DBR protocol. The transmission loss of DAE is 80 while AMCTD and DBR losing is between 110 to 120 .

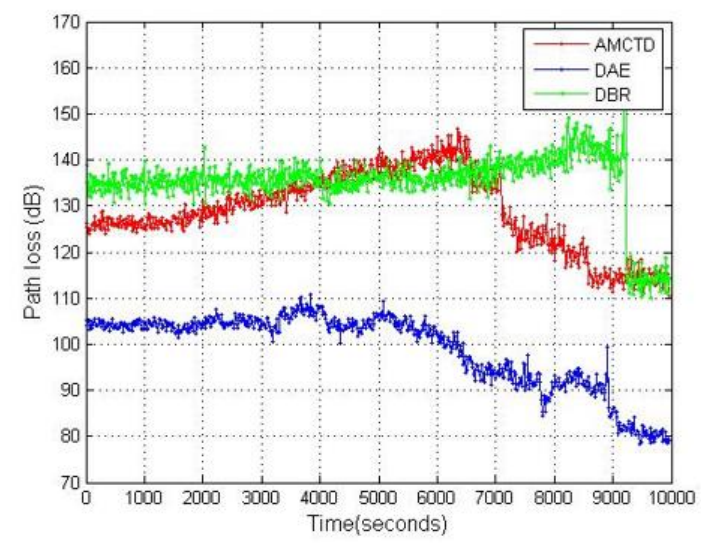

Figure 5: Transmission Loss vs Time

\section{Conclusion \& Future work}

In this paper we focused to increase the stability period and throughput as well as decreasing delay. We implemented 225 sensors in underwater environment with eight sinks. The main idea was to implement multiple sink nodes with the concept of Anycasting. In mathematical work, we have also addressed the channel conditions and formulated three different cost functions. These cost functions are derived using the various layers of water depth. These cost functions are totally dependent on the path-loss occurring in the dense underwater environment. In the final stage, we have compared our protocol DAE with two existing protocols DBR and AMCTD. Simulation results shows that DAE is more efficient than the previous schemes in stability period, end-to-end delay and throughput. In future, our focus will be on energy consumption. Keeping energy consumption in mind we will implement energy harvesting concept.

\section{References}

1. Wahid, Abdul, and Dongkyun Kim. "An energy efficient localization-free routing protocol for underwater wireless sensor networks." International journal of distributed sensor networks 8, no. 4 (2012): 307246

2. Bharamagoudra, Manjula R., and Bilal Gonen. "Event driven energy depth and channel aware routing for underwater acoustic sensor networks: Agent oriented clustering based approach." Computers \& Electrical Engineering 58 (2017): 1-19.

3. Kim, Hee-won, and Ho-Shin Cho. "SOUNET: SelfOrganized Underwater Wireless Sensor Network." Sensors 17, no. 2 (2017): 0283.

4. Javaid, Nadeem, Mohsin Raza Jafri, Zahoor Ali Khan, Umar Qasim, Turki Ali Alghamdi, and Muhammad Ali. "Iamctd: Improved adaptive mobility of courier nodes in threshold-optimized dbr protocol for underwater wireless sensor networks." International Journal of Distributed Sensor Networks (2014).

5. Javaid, Nadeem, Mehreen Shah, Ashfaq Ahmad, Muhammad Imran, and Athanasios V. Vasilakos. "An Enhanced Energy Balanced Data Transmission Protocol for Underwater Acoustic Sensor Networks." Sensors 16, no. 4 (2016): 487

6. Ahmed, Shiraz, N. Javaid, F. A. Khan, M. Y. Durrani, A. Ali, A. Shaukat, M. M. Sandhu, Z. A. Khan, and U. Qasim. "Co-UWSN: cooperative energy-efficient protocol for underwater WSNs." International Journal of Distributed Sensor Networks 11, no. 4 (2015): 891410.

7. Ilyas, Naveed, Nadeem Javaid, Zafar Iqbal, Muhammad Imran, Zahoor Ali Khan, Umar Qasim, and Muhammad Shoaib. "AAEERP: Advanced AUV-Aided Energy Efficient Routing Protocol for Underwater WSNs." In Advanced Information Networking and Applications (AINA), 2015 IEEE 29th International Conference on, pp. 77-83. IEEE, 2015.

8. Babu, A. V., and Susan Joshy. "Maximizing the data transmission rate of a cooperative relay system in an underwater acoustic channel." International Journal of Communication Systems 25, no. 2 (2012): 231-253.

9. Chao, Chih-Min, and Wei-Che Li. "DRP: An energyefficient routing protocol for underwater sensor networks." International Journal of Communication Systems (2017).

10. Ahmed, Sheeraz, Nadeem Javaid, Ashfaq Ahmad, Imran Ahmed, Mehr Yahya Durrani, Armughan Ali, Syed Bilal Haider, and Manzoor Ilahi. "SPARCO: stochastic performance analysis with reliability and cooperation for underwater wireless sensor networks." Journal of Sensors 2016 (2016)

11. Heinzelman, W. R., Chandrakasan, A., and Balakrishnan, H., "Energy-efficient communication protocol for wireless microsensor networks", In System Sciences: Proceedings of 
the 33rd Annual Hawaii International Conference, pp. 10pp, 2000.

12. Smaragdakis, G., Matta, I., and Bestavros, A.,"SEP: A Stable Election Protocol for clustered heterogeneous wireless sensor networks", Boston University Comp Science Department, 2004.

13. Qing, L., Zhu, Q., and Wang, M.’Design of a distributed energy-efficient clustering algorithm for heterogeneous wireless sensor networks", Computer communications, vol. 29, no. 12, pp. 2230-2237, 2006.

14. Y. Yun and Y. Xia, "Maximizing the Lifetime of Wireless Sensor Networks with Mobile Sink in Delay-Tolerant Applications", IEEE Transactions on Mobile Computing, vol. 9, no. 9, pp. 13081318, 2010.

15. Akbar, M., Javaid, N., Khan, A. A., Khan, Z. A., and Qasim, U., "On modeling geometric joint sink mobility with delay-tolerant cluster-less Wireless Sensor Networks", In Smart Communications in Network Technologies (SaCoNeT): IEEE International Conference, vol. 1, pp. 1-5, 2013.

16. J. Luo and J. P. Hubaux, "Joint Sink Mobility and Routing to Maximize the Lifetime of Wireless Sensor Networks: The Case of Constrained Mobility", IEEE/ACM Transactions on Networking, vol. 18, no. 3, pp. 871884, 2010.

17. W. Alsalih, H. Hassanein, and S. Akl, "Placement of multiple mobile data collectors in wireless sensor networks", Ad Hoc Networks, vol. 8, no. 4, pp. 378-390, 2010.

18. K. Singh and T. P. Sharma, "REDD: Reliable EnergyEfficient Data Dissemination in Wireless Sensor Networks with Multiple Mobile Sinks", International Science Index, vol: 7, no: 6, 2013.

19. Kinalis, A., Nikoletseas, S., Patroumpa, D., and Rolim, J., "Biased sink mobility with adaptive stop times for low latency data collection in sensor networks", Information Fusion, vol: 15, pp. 56-63, 2014. 